\title{
Article
}

\section{Forecasting Solar Energetic Particle Fluence with Multi-Spacecraft Observations}

Laitinen, Timo Lauri mikael, Dalla, Silvia, Battarbee, Markus and Marsh, M.S.

Available at http://clok.uclan.ac.uk/22888/

Laitinen, Timo Lauri mikael ORCID: 0000-0002-7719-7783, Dalla, Silvia ORCID: 0000-0002-7837-5780, Battarbee, Markus ORCID: 0000-0001-7055-551X and Marsh, M.S. (2018) Forecasting Solar Energetic Particle Fluence with MultiSpacecraft Observations. Proceedings of IAU Symposium 335: Space Weather of the Heliosphere: Processes and Forecasts, 13 (s335). pp. 298-300. ISSN $1743-9213$

It is advisable to refer to the publisher's version if you intend to cite from the work. http://dx.doi.org/10.1017/S1743921317008936

For more information about UCLan's research in this area go to http://www.uclan.ac.uk/researchgroups/ and search for < name of research Group>.

For information about Research generally at UCLan please go to http://www.uclan.ac.uk/research/

All outputs in CLoK are protected by Intellectual Property Rights law, including Copyright law. Copyright, IPR and Moral Rights for the works on this site are retained by the individual authors and/or other copyright owners. Terms and conditions for use of this material are defined in the policies page.

\section{CLoK}

Central Lancashire online Knowledge www.clok.uclan.ac.uk

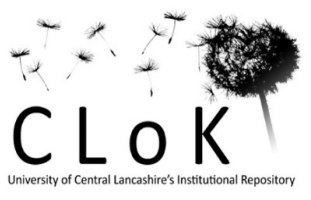




\title{
Forecasting Solar Energetic Particle Fluence with Multi-Spacecraft Observations
}

\author{
T. Laitinen ${ }^{1}$, S. Dalla ${ }^{1}$, M. Battarbee ${ }^{1}$ and M. S. Marsh ${ }^{2}$ \\ ${ }^{1}$ Jeremiah Horrocks Institute, University of Central Lancashire, Preston, United Kingdom. \\ email: tlmlaitinen@uclan.ac.uk \\ ${ }^{2}$ Met Office, Exeter, UK
}

\begin{abstract}
Forecasting Solar Energetic Particle (SEP) fluence, as integrated over an SEP event, is an important element when estimating the effect of solar eruptions on humans and technology in space. Current real-time estimates are based on SEP measurements at a single location in space. However, the interplanetary magnetic field corotates with the Sun approximately $13^{\circ}$ each day with respect to Earth, thus in 4 days a near-Earth spacecraft will have changed their connection about $60^{\circ}$ from the original SEP source. We estimate the effect of the corotation on particle fluence using a simple particle transport model, and show that ignoring corotation can cause up to an order of magnitude error in fluence estimations, depending on the interplanetary particle transport conditions. We compare the model predictions with STEREO observations of SEP events.
\end{abstract}

Keywords. Sun: Particle Emission, interplanetary medium, diffusion

\section{Introduction}

Total dose due to Solar Energetic Particles (SEPs) is an important factor in evaluation of radiation risk for astronauts and spacecraft. SEP fluence is affected by acceleration at the Sun and in interplanetary space (e.g. Reames 1999), scattering of particles along and across the field lines (e.g. Jokipii 1966), cross-field propagation due to field-line meandering (Laitinen et al. 2016) and drifts (Dalla et al. 2013), and motion of the observer relative to the SEP source region, i.e. corotation (Giacalone \& Jokipii 2012 Marsh et al. 2015). We study the effects of corotation on SEPs using a simple model and STEREO/LET (Mewaldt et al. 2008) SEP observations in 2013, when the spacecraft were at $\sim 60^{\circ}$ heliolongitudinal distance from each other. Such separation mimics the recently suggested L1/L5 mission concepts (e.g. Trichas et al. 2015).

\section{Effect of corotation of the SEP source: simple model}

We estimate the effect of the corotation of the particle source on the SEP intensities with a simple 1D diffusion model. We use impulsive injection with Gaussian longitudinal injection distribution, which gives the particle intensity $n$ at the observing spacecraft as

$$
n(t, r, \phi)=n_{0}(\phi) \frac{1}{2 \pi(\lambda v t / 3)^{3 / 2}} \mathrm{e}^{-\frac{3 r^{2}}{4 \lambda v t}}, \quad n_{0}(\phi)=n_{00} \mathrm{e}^{-(\Delta \phi)^{2} /\left(2 \sigma_{\phi}^{2}\right)}
$$

where $\phi$ and $r$ are the observer's heliographic longitude and radial distance from the Sun, $t$ is time from injection, $\lambda$ the scattering mean free path, $v$ the SEP speed, $\Delta \phi=$ $\phi_{F}-\phi_{S / C, F}$ with $\phi_{F}$ and $\phi_{S / C, F}$ the SEP source and spacecraft footpoint longitudes, $\sigma_{\phi}=40^{\circ}$ (e.g. Richardson et al. 2014), and $n_{00}$ a constant. We show in Figure 1 (a) the SEP intensities at $1 \mathrm{AU}$ for different constant $\Delta \phi$ values. 

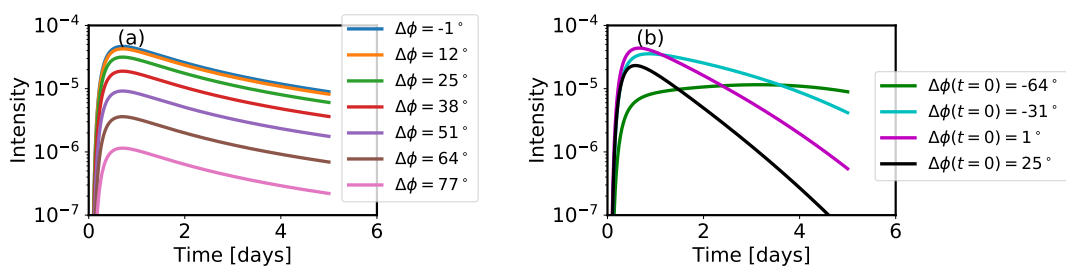

Figure 1. Time-intensity curves for $10 \mathrm{MeV}$ protons given by Eq. 2.1 with $\lambda=0.03 \mathrm{AU}$ at $r=1 \mathrm{AU}$. (a): SEP intensities on field lines that corotate with the Sun. (b): SEP intensities observed with spacecraft at $1 \mathrm{AU}$ moving relative to the corotating field lines.
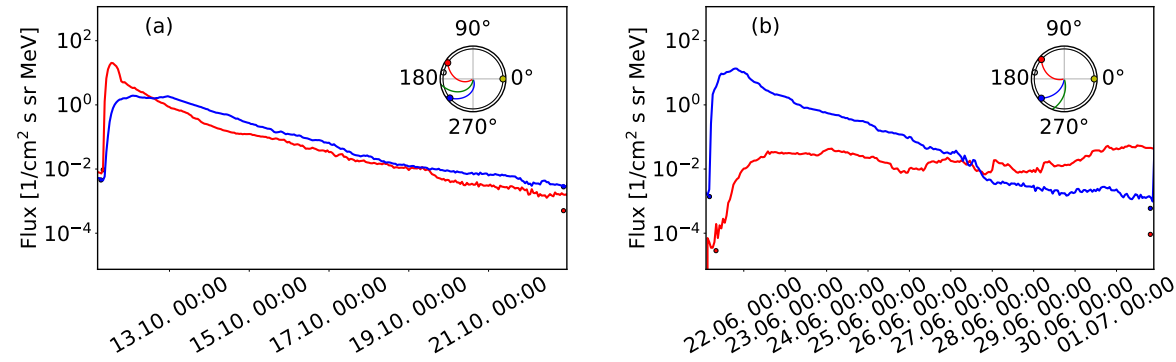

Figure 2. $10 \mathrm{MeV}$ proton intensities on (a) 11 October and (b) 21 June 2013. In the inset, the red and blue circles show the STA and STB locations at the start of the event, respectively, and the red, blue and green spirals show the Parker spirals connected to the STA, STB and the SEP source location.

A spacecraft observing the SEPs at $1 \mathrm{AU}$ rotates at rate $\Omega=-13.2^{\circ}$ day with respect to the SEP surface, giving $\Delta \phi=\phi_{F}-\left(\phi_{S / C, F}(t=0)+\Omega t\right)$. The spacecraft thus moves with respect to field lines that are connected to different-strength SEP injections at the Sun. The effect of this motion on SEP fluxes is demonstrated in Figure 1 (b), where the initially well-connected spacecraft (magenta curve) sees rapid SEP intensity onset followed by fast decay, whereas a spacecraft seeing the SEP event as central (green curve) sees the SEP intensities remaining high for several days. Similar features can be seen in SEP event profiles observed with different initial longitudinal separations (Cane et al. 1988). As seen in our simple model, corotation can produce these features without requiring a continuous, moving SEP source, as shown also by Marsh et al. (2015).

\section{Multi-spacecraft observations}

We analysed $10 \mathrm{MeV}$ proton intensities during 10 events where SEPs were observed by both STEREO/A (STA) and STEREO/B (STB) LET instruments (Mewaldt et al. 2008) in 2013. The source longitude $\phi_{F}$ was analysed from STEREO and SOHO observations, and compared with previous studies (e.g. Richardson et al. 2014), while $\phi_{S / C, F}$ was obtained using the solar wind velocity at STA and STB.

Figure 2 (a) shows an event with the SEP source between the two spacecraft. As with all $(4 / 10)$ events with this connectivity, the slower increase at STB is qualitatively consistent with STB propagating towards higher-intensity field lines. Figure 2 (b) shows an event where both spacecraft are advancing away from the source region. STA reaches $\Delta \phi=180^{\circ}$ on 27 June, which is seen as an increase in intensities. Overall, the SEP event profiles showed signatures of SEP source corotation.

In Figure 3 (a), we show 5-day fluences of $10 \mathrm{MeV}$ protons, modelled with Eq. 2.1p, as a 

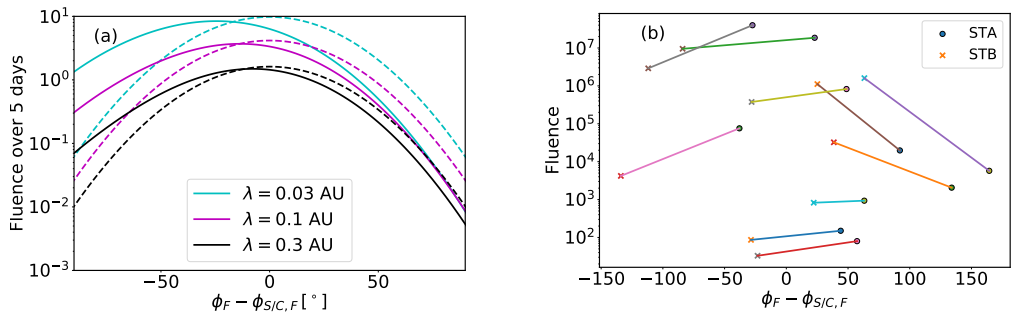

Figure 3. (a) Modelled $10 \mathrm{MeV}$ proton fluence at $1 \mathrm{AU}$ from the Sun as a function of $\Delta \phi(t=0)$. The dashed curves show the fluence if solar rotation is ignored. (b) Event-integrated fluences for $10 \mathrm{MeV} 10 \mathrm{SEP}$ events as a function of $\Delta \phi(t=0)$.

function of the initial $\Delta \phi$, both for cases including corotation (solid curves) and ignoring corotation (dashed curves). The corotation causes a significantly increased fluence for initially eastern SEP sources $(\Delta \phi(t=0)<0)$. The fluence also depends strongly on $\lambda$. In Figure 3 (b), we show the observed SEP fluences for the analysed events, as a function of the longitude. As can be seen, within a given event the fluences are generally larger for $\Delta \phi(t=0) \sim 0$, however there is considerable scatter, and low statistics prevent deducing clear asymmetries.

\section{Conclusions}

Propagation models show that the corotation of the SEP-filled field lines with the Sun affects the SEP intensities observed by interplanetary spacecraft. Our SEP event analysis shows that the time-intensity morphology is consistent with corotation affecting the event profiles. Low statistics prevent finding signatures of corotation in fluences without further, more involved modelling. Two-spacecraft observations bring more information, but require further study before their value can be estimated fully, including taking into account the varying interplanetary turbulence (e.g. Burlaga \& Turner 1976), which has significant influence on SEP transport along and across the mean field, and SEP fluences.

Acknowledgements. We acknowledge the STEREO/IMPACT, STEREO/SECCHI and SOHO/LASCO teams for providing the data used in this study. TL and SD acknowledge support from the UK Science and Technology Facilities Council (STFC) (grants ST/J001341/1 and ST/M00760X/1) and MB from the Leverhulme Trust Grant RPG2015-094.

\section{References}

Burlaga, L. F. \& Turner, J. M. 1976, JGR, 81, 73

Cane, H. V., Reames, D. V., \& von Rosenvinge, T. T. 1988, JGR, 93, 9555

Dalla, S., Marsh, M. S., Kelly, J., \& Laitinen, T. 2013, J. Geophys. Res. (Space Physics), 118, 5979

Giacalone, J. \& Jokipii, J. R. 2012, ApJL, 751, L33

Jokipii, J. R. 1966, ApJ, 146, 480

Laitinen, T., Kopp, A., Effenberger, F., Dalla, S., \& Marsh, M. S. 2016, A\&A, 591

Marsh, M. S., Dalla, S., Dierckxsens, M., Laitinen, T., \& Crosby, N. B. 2015, Space Weather, 13,386

Mewaldt, R. A., Cohen, C. M. S., Cook, W. R., et al. 2008, Space Sci. Rev., 136, 285

Reames, D. V. 1999, Space Sci. Rev., 90, 413

Richardson, I. G., von Rosenvinge, T. T., Cane, H. V., et al. 2014, Sol. Phys., 289, 3059

Trichas, M., Gibbs, M., Harrison, R., et al. 2015, Hipparchos, vol. 2, Issue 12, pp. 25 - 31, 2, 25 